\title{
Hypertriglyceridemia-Induced Pancreatitis With Rapid Response to Insulin Therapy
}

\author{
Sara Soliman
}

\begin{abstract}
Acute pancreatitis (AP) is one of the most common gastrointestinalrelated causes of hospitalization in the USA, accounting for more than 200,000 admissions annually. Although mild and moderate cases usually improve within a week, severe AP conditions could lead to lifethreatening pancreatic necrosis, multiple-organ dysfunction, and be fatal in some cases. Excessive alcohol use and gallstones are the two leading causes, nonetheless other systemic complication could also lead to AP. Hypertriglyceridemia is an important, yet uncommon, cause/risk factor of AP, especially when associated with heavy alcohol use. Additionally, the level of triglycerides (TGs) was found to be an important factor of determining the method and duration of treatment. Here we present a case of 38-year-old obese and active smoker male with hypertension and alcohol use disorder presented with a chief complaint of 2 weeks of progressive sharp epigastric pain. His medical history was significant of opioid use disorder that is maintained on methadone therapy. Computed tomography (CT) scan of the abdomen and pelvis revealed infiltration of the fat along the pancreatic tail and distal body with focus of decreased enhancement in the very distal pancreatic tail, which could represent a small infarct or phlegmon. In addition, laboratory data was significant of elevated lipase level $(>1,000 \mathrm{mg} / \mathrm{dL})$, which together with the CT result confirmed the diagnosis of AP. Additional laboratory workup revealed extremely high level of TGs of $>$ $2,000 \mathrm{mg} / \mathrm{dL}$. The patient was subsequently transferred to the intensive care unit for management of hypertriglyceridemia. He was started on insulin therapy along with supportive treatment for the management of pancreatitis. Hypertriglyceridemia and pancreatitis rapidly improved over the course of hospitalization period and no additional intervention was needed. He was successfully discharged on fenofibrate.
\end{abstract}

Keywords: Acute pancreatitis; Hypertriglyceridemia; Insulin; Phlegmon

\section{Introduction}

Acute pancreatitis (AP) is a life-threatening condition of pan-

Manuscript submitted September 25, 2020, accepted October 13, 2020

Published online November 18, 2020

Yale-Waterbury Internal Medicine Residency Program, Waterbury Hospital, 64 Robbins St., Waterbury, CT 06708, USA.Email: saraelhakim7@gmail.com

doi: https://doi.org/10.14740/jmc3595 creatic inflammation, with an annual incidence of 20 - 40 per 100,000 population in the USA [1]. Although the majority of AP cases are self-limited and resolve within a week, severe AP is an emergency condition due to the risk of developing local complications (necrosis, pseudocyst, phlegmon), or systemic multiorgan failure [2]. The severity of AP is classified based on well-established criteria and scoring systems. In particular, the Atlanta classification identifies three categories of AP, mild, moderate or severe [3]. Mild AP resolves in the first week and does not involve local or systemic complications. Moderately acute is recognized by transient organ failure and local complication or exacerbation of co-morbid disease. Severe acute $\mathrm{AP}$ is characterized by permeant organ failure $(>48 \mathrm{~h})$. In addition, bedside index for severity in AP (BISAP) identify patients at high risk of mortality during the early phase of disease, with scores ranging from 0 - 2 in lower-risk and $3-5$ in higher-risk cases [4].

Recently, hypertriglyceridemia received an attention as a potential cause of AP and a strong cofactor, when associated with other well-established etiologies, such as heavy alcohol consumption and gallstones [5, 6]. Hypertriglyceridemia-induced pancreatitis cases were found to have higher incidence of complications and organ failures, as well as more severe hospital course [6]. The typical treatment plan of hypertriglyceridemia involves omega-3-fatty acids, however in severe cases, insulin therapy was shown to be a successful procedure, especially in cases resulting in AP [5]. In addition, plasmapheresis was shown to be a useful tool in the management of hypertriglyceridemia-induced pancreatitis [7]. Here we report a case of hypertriglyceridemia-induced pancreatitis in a 38-year-old male with a 20 -year history of excessive alcohol consumption. The case highlights the importance of considering hypertriglyceridemia in the diagnosis of AP cases with presentations pointing towards different etiologies. We will also review the pathogenesis and prognosis of hypertriglyceridemia-induced pancreatitis, with a specific focus on the medical options for management of the underlying hypertriglyceridemia.

\section{Case Report}

A 38-year-old male presents with progressive epigastric abdominal pain. His medical history was significant for opioid use disorder maintained on methadone, hypertension, past motor vehicle accident with residual chronic back pain. He reported heavy alcohol use with average of $3-10$ glasses of brandy daily for almost 20 years. He is obese with body mass 
index (BMI) of $31.5 \mathrm{~kg} / \mathrm{m}^{2}$, yet not diabetic and no history of gallstones or pancreatitis was noted. Family history was not significant for pancreatitis or hypertriglyceridemia.

On presentation, he described a progressive severe abdominal pain radiating to the back associated with nausea and foul-smelling diarrheal episodes. Physical examination was notable for diffuse tenderness to palpation without guarding or rigidity. His laboratory workup was notable for leukocytosis $\left(20.7 \times 10^{3} / \mathrm{mm}^{3}\right.$; reference: $\left.4.5-11 \times 10^{3} / \mathrm{mm}^{3}\right)$, with neutrophilic predominance of $82.5 \%$, but no bandemia. Platelets $189 \times 10^{3} / \mathrm{mm}^{3}$ (reference: $150-400 \times 10^{3} / \mathrm{mm}^{3}$ ), sodium 134 $\mathrm{mmol} / \mathrm{L}$ (reference: 135 - $145 \mathrm{mmol} / \mathrm{L}$ ), creatinine $1.29 \mathrm{mg} / \mathrm{dL}$ (reference: $0.84-1.21 \mathrm{mg} / \mathrm{dL}$ ), amylase $145 \mathrm{U} / \mathrm{L}$ (reference: 30 - $110 \mathrm{U} / \mathrm{L}$ ), lipase 1,113 U/L (reference: < $160 \mathrm{U} / \mathrm{L}$ ), bilirubin $0.7 \mathrm{mg} / \mathrm{dL}$ (reference: $0.1-1.2 \mathrm{mg} / \mathrm{dL}$ ), aspartate aminotransferase (AST) $60 \mathrm{IU} / \mathrm{L}$ (reference: 7 - $56 \mathrm{IU} / \mathrm{L}$ ), alanine aminotransferase (ALT) $34 \mathrm{IU} / \mathrm{L}$ (reference: 29 - 33 IU/L), alkaline phosphatase $85 \mathrm{IU} / \mathrm{L}$ (reference: 44 - $147 \mathrm{IU} / \mathrm{L}$ ). Computed tomography (CT) scan of abdomen and pelvis showed infiltration of the fat along the pancreatic tail and distal body, suggestive of AP. Focus of decreased enhancement in the very distal pancreatic tail was noted and indicating a potential formation of phlegmon or small infarct (Fig. 1). BISAP score was 2 , indicating low risk of mortality of less than $2 \%$. Based on Atlanta classification, the condition was classified as moderately severe AP, evident by transit organ failure (acute kidney injury that lasted $<48 \mathrm{~h}$ ), systemic inflammation (leukocytosis), and possible local complications (infarct/phlegmon).

Initial management included conventional supportive treatment of pancreatitis with intensive fluid hydration, bowel rest, and pain management. He was also started on the Clinical Institute Withdrawal Assessment for Alcohol (CIWA) protocol for the assessment and management of alcohol withdrawal. The patient later developed a low-grade fever $\left(38.2^{\circ} \mathrm{C}\right)$, which was attributed to the ongoing systemic inflammation. At this point, no indication of developing necrosis or sepsis was noted, and therefore no antibiotics were included in the treatment plan. Additional laboratory workup revealed high triglycerides (TGs) level of $2,625 \mathrm{mg} / \mathrm{dL}$ (reference: $<150 \mathrm{mg} / \mathrm{dL}$ ). Thus, insulin therapy was started, and fluids were changed to dextrose to reduce the risk of hypoglycemia. TGs levels were markedly reduced with insulin therapy to $1,110 \mathrm{mg} / \mathrm{dL}$ in the first $12 \mathrm{~h}$, and then to 502 and $431 \mathrm{mg} / \mathrm{dL}$ in less than $48 \mathrm{~h}$. On day 6 in hospital, TGs level was restored to normal value at $150 \mathrm{mg} /$ dL. Due to the remarkable improvement in the TGs level and overall condition, additional intervention with plasmapheresis was not needed. Prior to discharge, the patient was counselled on the importance of dietary changes and the control of obesity and alcohol heavy consumption. Due to the potential of developing local/regional complication, such as pseudocyst, the patient was scheduled for follow-up CT scans in $4-6$ weeks. Finally, he was discharged on daily fenofibrate therapy with a plan for outpatient alcohol rehabilitation.

\section{Discussion}

Hypertriglyceridemia is the third most common cause of AP,

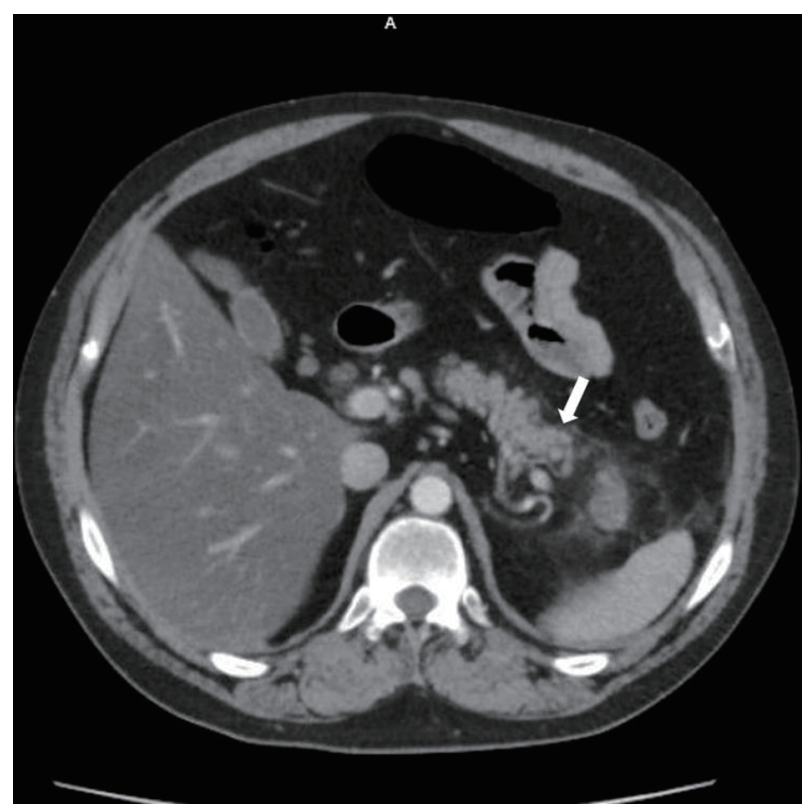

Figure 1. CT of the abdomen showing pancreatitis. White arrow denotes focus of decreased enhancement in the very distal pancreatic tail that may represent a phlegmon or infarct. CT: computed tomography.

with a prevalence approaching 10\% [8]. High level of TGs $(>2,000 \mathrm{mg} / \mathrm{dL})$ is associated with an increased risk of pancreatitis, reaching 10-20\% [9]. Early identification and rapid reduction of TGs level are crucial for management of hypertriglyceridemia-induced pancreatitis. Inherited dyslipidemia is the leading primary cause of hypertriglyceridemia-induced pancreatitis, however It also could originate from secondary causes, such as poorly controlled diabetes and certain medications [10]. Furthermore, heavy alcohol intake poses a risk of developing hypertriglyceridemia-induced pancreatitis.

The exact mechanism by which hypertriglyceridemia adds to the pathogenesis of AP remains controversial. The most prevalent mechanism highlights the release of fatty acids from the hydrolysis of TG rich proteins or chylomicrons. High concentration of unsaturated fatty acids could result in pancreatic cell damage by inducing a proinflammatory response, calcium release, and inhibiting mitochondrial complexes [11]. Damage of pancreatic acinar cells, platelets, and vascular endothelium induces acidosis and ischemia [12]. Metabolic acidosis further increases the toxicity of fatty acids. In another potential mechanism, chylomicrons increase plasma viscosity, which could lead to capillary plugging and ischemia [10].

Hypertriglyceridemia-induced pancreatitis is more common in obese male patients, with a notably higher prevalence in case of diabetes [9]. Additionally, obesity was found to be associated with more severe complications, including multiorgan failure [12]. The larger volume of intrapancreatic adipocytes increases the risk of extensive necrosis during attacks of AP. Therefore, management of fatty acid-induced lipotoxicity is key for the treatment of hypertriglyceridemia-induced pancreatitis, especially in obese humans.

Alcohol consumption increases the risk of hypertriglyceridemia and is perceived as well-established etiology of AP 
[12]. For instance, by competing for oxidation with free fatty acids, alcohol increases their availability for TGs synthesis and induces the secretion of very-low-density lipoprotein (VLDL) from the liver, which both predispose to hypertriglyceridemia and chylomicronemia [12]. Moreover, non-oxidative pancreatic metabolism of ethanol by fatty acid ethyl ester (FAEE) synthases induces the production of fatty acids, which also increase the likelihood of hyperlipidemia and pancreatitis [13].

As for the management of hypertriglyceridemia-induced pancreatitis, insulin with or without heparin, plasmapheresis, and hemofiltration are the commonly used therapy modalities [14]. Insulin is usually the first-line therapy for reducing TGs level, either subcutaneously delivered or through intravenous infusion [14]. Continuous insulin infusion is given until TGs level drops to $<500 \mathrm{mg} / \mathrm{dL}$ even if blood glucose levels are in the normal range [14]. Insulin lowers TGs level by suppressing hormone-sensitive lipase in adipocytes, the key enzyme for breaking down adipocyte TGs into free fatty acids. In addition, insulin stimulates the synthesis of lipoprotein lipase (LPL), which induces the metabolism of chylomicron and VLDL to glycerol and free fatty acids [15]. Heparin activates LPL and can be used in combination with insulin. After the initial increase in LPL activity, heparin induces a quick hepatic degradation of the enzyme and causes depletion of plasma LPL and could result in further increases of chylomicrons [16]. Therefore, the effect of heparin is usually transient and should only be given for a short time as an initial procedure in the treatment plan [16].

Plasmapheresis is another treatment option for quick and effective reduction of TGs level, however cost-effectiveness, staffing resources, and complications are concerns that limit the wide use of plasmapheresis in the treatment of hypertriglyceridemia [17].The question whether insulin is inferior to plasmapheresis for management of hypertriglyceridemia-induced AP is being addressed in an ongoing multicenter controlled trial (BI-TPAI) [14]. The study aims to conclude that intensive insulin therapy is not inferior to plasmapheresis and can be an effective, cheaper, and safe therapy. In a retrospective study, both intravenous insulin and plasmapheresis showed a substantial decrease in TGs level, but no difference in clearance rate or length of stay was noted [18]. Collectively, these studies suggest that insulin therapy can rapidly reduce TGs level and is a safer and more cost-effective modality compared to plasmapheresis in the management of hypertriglyceridemiainduced pancreatitis.

\section{Conclusions}

Hypertriglyceridemia accounts for up to $10 \%$ of AP cases. Early recognition and diagnosis are important for selecting the appropriate management plan. We presented a case of hypertriglyceridemia-induced pancreatitis in a 38-year-old male with a history of alcohol and opioid use. While alcohol consumption initially appeared to be the cause of AP, laboratory data revealed an underlying severe hypertriglyceridemia, which has been the main focus of the treatment plan. The case emphasizes the importance of considering hypertriglyceridemia as the underlying cause of AP, especially in patients with a history of alcohol consumption or other common etiologies. There are several mechanisms explaining how hypertriglyceridemia leads to AP and how alcohol consumption contributes to the pathological process of hypertriglyceridemia. In case of hypertriglyceridemia-induced pancreatitis, rapid reduction of TGs level is of paramount importance for successful management of the case. In the majority of hypertriglyceridemia-induced pancreatitis cases, plasmapheresis can be replaced by an intensive intravenous insulin course, which proved itself as an effective and safe alternative therapy with rapid improvement in the TGs level and overall AP condition.

\section{Acknowledgments}

The author would like to thank Dr. Medhat Ghaly at YaleWaterbury Internal Medicine Residency Program for helpful discussions and critical reading of the manuscript.

\section{Financial Disclosure}

None to declare.

\section{Conflict of Interest}

None to declare.

\section{Informed Consent}

Not applicable.

\section{Author Contributions}

SS conceived the study, reviewed literature, and wrote the manuscript.

\section{Data Availability}

All data that support the findings of the study are available from the author on request.

\section{References}

1. Yadav D, Lowenfels AB. The epidemiology of pancreatitis and pancreatic cancer. Gastroenterology. 2013;144(6):12521261.

2. Greenberg JA, Hsu J, Bawazeer M, Marshall J, Friedrich JO, Nathens A, Coburn N, et al. Clinical practice guideline: management of acute pancreatitis. Can J Surg. 2016;59(2):128-140.

3. Thoeni RF. The revised Atlanta classification of acute 
pancreatitis: its importance for the radiologist and its effect on treatment. Radiology. 2012;262(3):751-764.

4. Gao W, Yang HX, Ma CE. The value of BISAP score for predicting mortality and severity in acute pancreatitis: a systematic review and meta-analysis. PLoS One. 2015;10(6):e0130412.

5. Khan R, Jehangir W, Regeti K, Yousif A. Hypertriglyceridemia-induced pancreatitis: choice of treatment. Gastroenterology Res. 2015;8(3-4):234-236.

6. Garg R, Rustagi T. Management of hypertriglyceridemia induced acute pancreatitis. Biomed Res Int. 2018;2018:4721357.

7. Joglekar K, Brannick B, Kadaria D, Sodhi A. Therapeutic plasmapheresis for hypertriglyceridemia-associated acute pancreatitis: case series and review of the literature. Ther Adv Endocrinol Metab. 2017;8(4):59-65.

8. Tsuang W, Navaneethan U, Ruiz L, Palascak JB, Gelrud A. Hypertriglyceridemic pancreatitis: presentation and management. Am J Gastroenterol. 2009;104(4):984-991.

9. Nawaz H, Koutroumpakis E, Easler J, Slivka A, Whitcomb DC, Singh VP, Yadav D, et al. Elevated serum triglycerides are independently associated with persistent organ failure in acute pancreatitis. Am J Gastroenterol. 2015;110(10):1497-1503.

10. de Pretis N, Amodio A, Frulloni L. Hypertriglyceridemic pancreatitis: Epidemiology, pathophysiology and clinical management. United European Gastroenterol J. 2018;6(5):649-655.

11. Yang F, Wang Y, Sternfeld L, Rodriguez JA, Ross C, Hayden MR, Carriere F, et al. The role of free fatty acids, pancreatic lipase and $\mathrm{Ca}+$ signalling in injury of isolated acinar cells and pancreatitis model in lipoprotein lipasedeficient mice. Acta Physiol (Oxf). 2009;195(1):13-28.

12. Navina S, Acharya C, DeLany JP, Orlichenko LS, Baty CJ, Shiva SS, Durgampudi C, et al. Lipotoxicity causes multisystem organ failure and exacerbates acute pancreatitis in obesity. Sci Transl Med. 2011;3(107):107ra110.

13. Clemens DL, Schneider KJ, Arkfeld CK, Grode JR, Wells MA, Singh S. Alcoholic pancreatitis: New insights into the pathogenesis and treatment. World J Gastrointest Pathophysiol. 2016;7(1):48-58.

14. Song X, Shi D, Cui Q, Yu S, Yang J, Song P, Walline J, et al. Intensive insulin therapy versus plasmapheresis in the management of hypertriglyceridemia-induced acute pancreatitis (Bi-TPAI trial): study protocol for a randomized controlled trial. Trials. 2019;20(1):365.

15. Eckel RH. Lipoprotein lipase. A multifunctional enzyme relevant to common metabolic diseases. N Engl J Med. 1989;320(16):1060-1068.

16. Korn ED. Clearing factor, a heparin-activated lipoprotein lipase. I. Isolation and characterization of the enzyme from normal rat heart. J Biol Chem. 1955;215(1):1-14.

17. Sadur CN, Eckel RH. Insulin stimulation of adipose tissue lipoprotein lipase. Use of the euglycemic clamp technique. J Clin Invest. 1982;69(5):1119-1125.

18. Tamez-Perez HE, Saenz-Gallegos R, Hernandez-Rodriguez K, Forsbach-Sanchez G, Gomez-de Ossio MD, Fernandez-Garza N, Zapata-de la Garza E, et al. [Insulin therapy in patients with severe hypertriglyceridemia]. Rev Med Inst Mex Seguro Soc. 2006;44(3):235-237. 